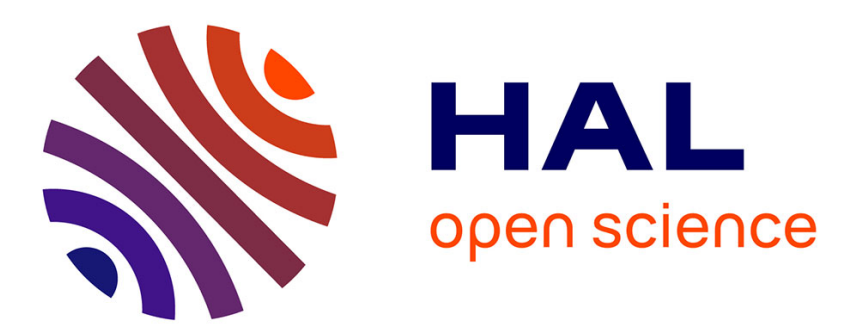

\title{
Nonlinear conductivity of a two-dimensional pinned charge-density wave in a strong magnetic field
}

P. Littlewood, A. Millis, X. Zhu, Bernard Normand

\section{To cite this version:}

P. Littlewood, A. Millis, X. Zhu, Bernard Normand. Nonlinear conductivity of a two-dimensional pinned charge-density wave in a strong magnetic field. Journal de Physique IV Proceedings, 1993, 03 (C2), pp.C2-9-C2-14. 10.1051/jp4:1993202 . jpa-00251571

\section{HAL Id: jpa-00251571 https://hal.science/jpa-00251571}

Submitted on 1 Jan 1993

HAL is a multi-disciplinary open access archive for the deposit and dissemination of scientific research documents, whether they are published or not. The documents may come from teaching and research institutions in France or abroad, or from public or private research centers.
L'archive ouverte pluridisciplinaire HAL, est destinée au dépôt et à la diffusion de documents scientifiques de niveau recherche, publiés ou non, émanant des établissements d'enseignement et de recherche français ou étrangers, des laboratoires publics ou privés. 


\title{
Nonlinear conductivity of a two-dimensional pinned charge-density wave in a strong magnetic field
}

\author{
P.B. LITTLEWOOD, A.J. MILLIS, X. ZHU and B.G.A. NORMAND* \\ AT \& T Bell Laboratories, Murray Hill, NJ 07974, U.S.A. \\ ${ }^{*}$ Department of Physics, M.I.T., Cambridge, MA 02139, U.S.A.
}

\begin{abstract}
We present the theory of the dynamics of a two-dimensional Wigner crystal in a high magnetic field, assuming charge-density-wave correlations extend over a distance large compared to the interelectronic spacing. We consider the effects of both weak disorder and screening by uncondensed carriers. In an electric field large compared to the threshold field for sliding, a perturbative theory is used to calculate the nonlinear conductivity and correlation functions of the elastic strain. We compare our results to the behavior of conventional charge-density-wave systems, and stress the role of the large magnetic field, soft shear modulus, and screening by the background linear conductivity.
\end{abstract}

\section{Introduction}

In a sufficiently high magnetic field, the two-dimensional electron system (2DES) is expected to form a Wigner crystal(WC). Observation of diffraction peaks caused by charge ordering would be unambiguous evidence for the WC, but these are expected to be prohibitively difficult to see. Consequently, experimental efforts to elucidate the behavior of the putative WC state have focussed on another characteristic of the crystal, namely the collective aspects of the transport.

Around $\nu=1 / 5$, insulating phases have been observed in the cleanest samples currently available [1, 2, 3, 4, 5], and nonlinearities in the d.c. conductivity have been reported by several groups $[2,3,4,5]$. The temperaturedependent onset of the nonlinearity has been associated with the collective sliding motion of the Wigner crystal. Microwave absorption[3, 6], surface acoustic wave [7] and RF absorption[8] measurements of the (assumed linear) a.c. response have been interpreted as evidence for a pinned (or dispersing) shear mode in the solid. Both types of experiments are consistent with the notion of an elastic crystal pinned by the inevitable disorder, and are broadly similar to the phenomena observed in pinned charge-density wave (CDW) systems [9] or flux lattices (FL) in type II superconductors [10].

In this paper, we discuss the simplest theoretical model of the pinned WC - a classical elastic medium interacting with a weak local random potential in a large magnetic field. For reasons of space, we quote the principal results and details of the calculations can be found elsewhere [11, 12]. We shall focus here on a physical motivation of the behavior, discuss the similarities and differences with pinned CDW's and flux lattices (FL), and outline the conditions under which our simple model is expected to be valid.

\section{Model}

We treat the Wigner crystal as a two-dimensional elastic continuum characterized by charge and mass densities $\rho_{c}$ and $\rho_{m}$ and shear and bulk elastic constants $\mu$ and $\lambda .[13]$ These are given in terms of the lattice constant $a$ by classical electrostatics[14, 15] as

$$
\lambda=\alpha_{\lambda} \rho_{c}^{2} a / \epsilon, \text { and, } \mu=\alpha_{\mu} \rho_{c}^{2} a / \epsilon
$$

Here $\epsilon$ is the dielectric constant of the host lattice, and we shall use the values $\alpha_{\lambda} \approx 1, \alpha_{\mu} \equiv \alpha \approx 0.02$ appropriate for a classical triangular lattice. The equation of motion in real space the two-dimensional displacement $\vec{u}(\vec{r}, t)$ of the WC at the point $(\vec{r}, t)$ is then

$$
\rho_{m} \ddot{\vec{u}}+\gamma \rho_{m} \dot{\vec{u}}+\rho_{m} \omega_{c} \vec{e}_{z} \times \dot{\vec{u}}+\int D\left(\vec{r}-\overrightarrow{r^{\prime}}\right) \cdot \vec{u}\left(\overrightarrow{r^{\prime}}, t\right) d^{2} \overrightarrow{r^{\prime}}=\rho_{c} \vec{E}^{e x t}-\rho(\vec{r}) \nabla_{\vec{r}} V_{d i s}(\vec{r}+\vec{u}(\vec{r}, t)) .
$$


This equation includes the inertial response, a phenomenological damping constant $\gamma$, the Lorentz force (with the cyclotron frequency $\left.\omega_{c}=\rho_{c} B / \rho_{m} c\right)$, the elastic dynamical matrix $D$, and coupling to the disorder potential $V_{d i s}(\vec{r})$. We shall include in the dynamical matrix the long-range Coulomb interaction between density fluctuations of the lattice, mediated by the "background" response of the material. This we assume to include not only the dielectric response of the medium but also conduction by "free" or thermally excited carriers, not directly part of the condensate. We shall incorporate the effects of the carriers via the measured $q-$ and $\omega-$ dependent linear conductivity.[11] $\vec{E}^{e x t}$ is the applied electric field. The spatially varying charge density is related to the charge-density wave order-parameter in reciprocal $(\vec{G})$-space $\Delta(\vec{G})$ as: $\rho(\vec{r})=\sum_{\vec{G}} \Delta(\vec{G}) e^{i \vec{G} \cdot \vec{r}}$.

It is convenient to separate the the displacement vector into longitudinal $\left(u_{L}\right)$ and transverse $\left(u_{T}\right)$ parts, and then the equation of motion in $\vec{q}, \omega$ space is

$$
\left(\begin{array}{cc}
D_{L} & -i \omega \omega_{c} \\
\dot{i} \omega \omega_{c} & D_{T}
\end{array}\right)\left(\begin{array}{l}
u_{L} \\
u_{T}
\end{array}\right)=\frac{\rho_{c}}{\rho_{m}}\left[\left(\begin{array}{c}
E_{L} \\
E_{T}
\end{array}\right)-\left(\begin{array}{c}
E_{L}^{P} \\
E_{T}^{P}
\end{array}\right)\right]
$$

Here we have written the pinning force as an equivalent field $\vec{E}^{P}$ - it is of course non-linearly dependent on the displacement vector $\vec{u}$. The transverse and longitudinal dynamical matrices are

$$
D_{L, T}=-\omega^{2}+i \gamma \omega+\omega_{L, T}^{2}
$$

with

$$
\omega_{T}^{2}=\alpha \Omega^{2}(g a)^{2}
$$

and

$$
\omega_{L}^{2}=\Omega^{2}\left[(1+\alpha)(q a)^{2}+\frac{2 \pi q a}{1-\left(i q a \sigma_{x x} / \sigma_{o}\right) /(\omega / \Omega)}\right]
$$

Here we have defined a characteristic frequency scale $\Omega^{2}=\rho_{c}^{2} / \rho_{m} a \epsilon$ and a scale for the conductivity $\sigma_{0}=a \epsilon \Omega / 2 \pi=$ $\left(e^{2} / \hbar\right) \times(\hbar n / 2 \pi m \Omega)$ Typical numbers for GaAs/GaAlAs inversion layers in the high field regime are $\omega_{c}=4 \times$ $10^{13} \mathrm{sec}^{-1}, \Omega=1.8 \times 10^{12} \mathrm{sec}^{-1}, \sigma_{0}=2 \times 10^{-5} \Omega^{-1}$ Notice that in the longitudinal response we have included the screened Coulomb interaction between density fluctuations of the WC, incorporating the dynamic response of the "uncondensed" carriers, modelled here by their conductivity $\sigma_{x x}$. In the limit $q, \omega$ and $q / \omega \rightarrow 0$, this is the measured linear conductivity [11]. If the conductivity is small enough to be neglected, Eq. (6) yields the usual two-dimensional plasmon dispersion $\omega_{L} \propto q^{\frac{1}{2}}$, but in the opposite limit this term leads to an effective damping term in the equation of motion Eq. (2).

$$
\gamma_{e f f} \rho_{m} \dot{u}_{L}=\frac{\rho_{c}^{2}}{\sigma_{x x}} \dot{u}_{L}
$$

We shall argue that this term is very important near threshold when the WC is sliding very slowly.

It is interesting to compare this model with the Fukuyama-Lee-Rice (FLR) [16] for sliding CDW's, and to note the differences. Firstly, the CDW model is of a scalar field $\phi=Q_{z} u(\vec{r}, t)$ since the CDW is assumed to move only in the chain- (z-) direction. There are only longitudinal fluctuations, which means that the long-range Coulomb force is always operative in coupling spatial fluctuations of $\phi$. In the WC, the transverse fluctuations are much softer than the longitudinal modes (since $\alpha \ll 1$ ); this means that in the static limit, purely transverse fluctuations can pin the WC to disorder, and the long-range Coulomb interaction can be neglected, even at $T=0$. At finite frequency, or in the sliding state, the longitudinal and transverse fluctuations of the WC are coupled by the large magnetic field. We are in the limit where $\omega_{c}$ is the largest frequency scale in the problem, so the Lorentz force is the dominant term in the equation of motion. Finally, of course, here we have an explicitly two-dimensional problem, which shows up most crucially in the $q^{\frac{1}{2}}-$ dependence of the plasmon.

A less obvious difference between the models relates to the underlying physics. In CDW's the amplitude of the density wave compared to the average charge density is small, whereas in the WC it is of order unity, except close to a possible second-order phase transition. Paradoxically, if the amplitude is small, the effect of impurities on the amplitude of the density wave is small; for a potential of strength $v$, the local suppression of the amplitude of the density wave is of order $\left(\rho_{c} v / W\right)(a / \xi)^{(d-1)}$ (here $W$ is the bandwidth, $\xi$ the coherence length and $d$ the dimension)[16]. Since $\xi$ is inversely proportional to the amplitude, CDW's naturally have weak pinning, in the sense that the amplitude of the CDW is only weakly suppressed by an impurity, and the dominant fluctuations are phase distortions on long length scales. There is no such expectation for the case of a Wigner crystal.

\section{Collective modes}

First, consider the case when pinning is neglected, and set $\vec{E}^{P}=0$. We also neglect the phenomenological damping term and take $\gamma=0$. Since $\omega_{c}$ is large, the solution of Eq. (3) is a mode at the cyclotron frequency, and a second dispersing mode

$$
\omega=\alpha^{\frac{3}{2}} \Omega(q a) \omega_{L}(q, \omega) / \omega_{c}
$$




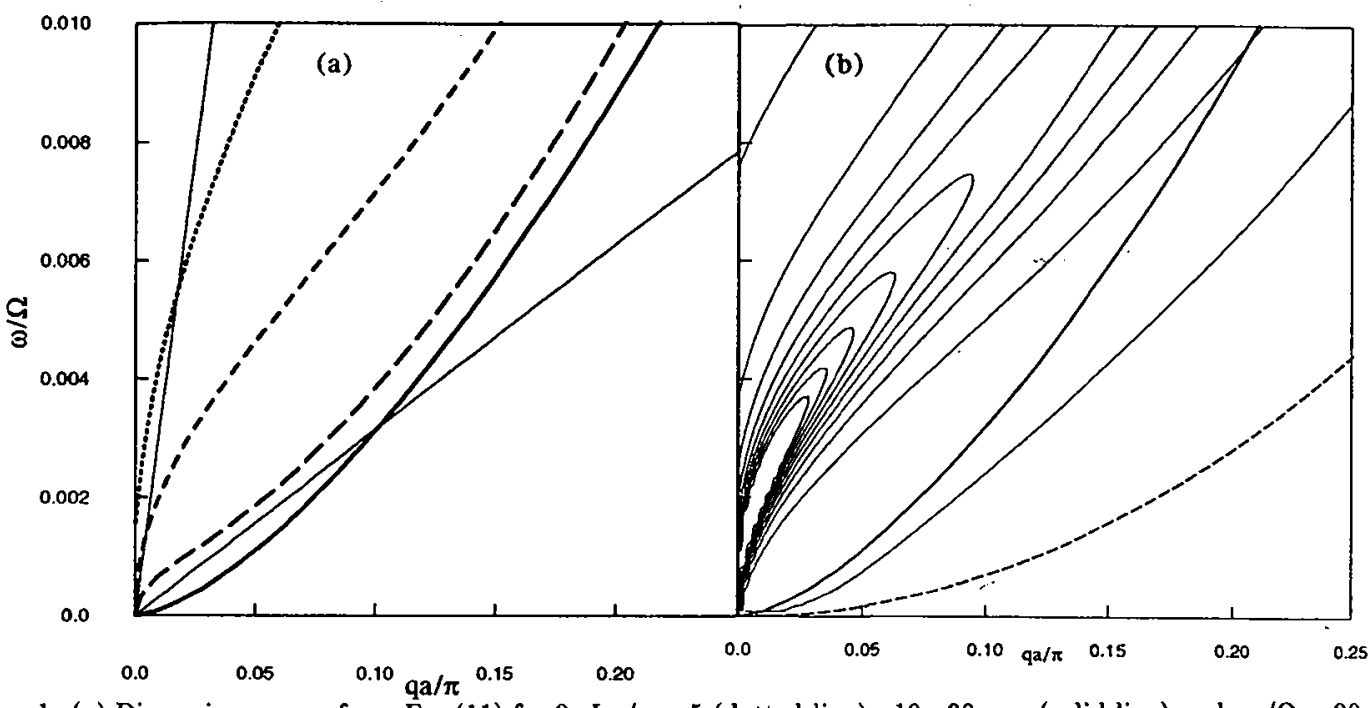

Figure 1: (a) Dispersion curves from Eq. (11) for $2 \pi L_{T} / a=5$ (dotted line) , $10,30, \infty$ (solid line) and $\omega_{c} / \Omega=20$. The thin straight lines mark $\omega / \Omega=\left(\sigma_{x x} / \sigma_{0}\right)(q a)$ for $\sigma_{x x} / \sigma_{0}=0.01,0.1$; for corresponding values of the conductivity the modes are overdamped below these lines. (b) Equally spaced contours of power dissipated in a longitudinal field, for $2 \pi L_{T} / a=10, \omega_{c} / \Omega=20$ and $\sigma_{x x} / \sigma_{0}=0.01$. The solid and dashed lines mark the dispersion of the $q^{3 / 2}$ and $q^{2}$ modes, respectively, in the absence of pinning. The width of the absorption feature at $q \rightarrow 0$ is produced by inhomogeneous broadening due to disorder. Note that significant power is absorbed in a longitudinal field only at frequencies large enough that $\omega / \Omega \gg\left(\sigma_{x x} / \sigma_{o}\right)(q a)$. (From [11])

The various regimes of dispersion are demarcated in Figure 1. From Eq. (6), we see that if $\omega / \Omega \gg\left(\sigma_{x x} / \sigma_{o}\right)(q a)$ an underdamped mode exists with dispersion

$$
\omega=(2 \pi \alpha)^{\frac{1}{2}} \frac{\Omega^{2}}{\omega_{c}}(q a)^{3 / 2}
$$

Consequently, an undamped shear mode can exist only for large $q$. In the limit $\omega / \Omega \ll\left(\sigma_{x x} / \sigma_{0}\right)(q a)$, the Coulomb term contributes to the damping of the mode, rather than the restoring force; in particular, if $\sigma_{x x} / \sigma_{0} \ll 2 \pi[\alpha /(1+$ $\alpha)^{\frac{1}{2}} \Omega / \omega_{c}$, then there is a quadratically dispersing underdamped mode, where the Wigner crystal and the uncondensed carriers oscillate in phase, with dispersion $\omega=[\alpha(1+\alpha)]^{\frac{1}{2}} \frac{\Omega^{2}}{\omega_{c}}(q a)^{2}$

At applied dc electric fields below threshold, the effect of the pinning is to distort the crystal into domains of characteristic linear dimension $L_{T}[10,11,16]$. Within each domain, the response to an ac field is an oscillation about the pinned position, with eigenvalues of order

$$
\bar{\omega}_{p}^{2}=\left[\omega_{T}\left(q=1 / L_{T}\right)\right]^{2}=\alpha \Omega^{2}\left(a / L_{T}\right)^{2}
$$

In fact, the distribution of these eigenvalues is as wide as the mean, so that any line will be inhomogeneously broadened. Nevertheless, one may crudely include the pinning as a uniform force in space, which then leads to a mode dispersion given by

$$
\omega^{2}=\left[\omega_{L}^{2}+\bar{\omega}_{p}^{2}\right]\left[\omega_{T}^{2}+\bar{\omega}_{p}^{2}\right] / \omega_{c}^{2}
$$

Some typical results are shown in Fig. 1. Again, there are different limits depending on the value of $\sigma_{x x}$. If this is negligible, Eq. (11) gives the usual $q^{3 / 2}$ dispersion for $q>L_{T}^{-1}$, but for a length scale $L_{T}^{-1}>q>L_{L}^{-1}$ we get a mode with a $2 \mathrm{D}$-plasmon dispersion[3] $\omega=\left(\Omega \bar{\omega}_{p} / \omega_{c}\right)(2 \pi q a)^{\frac{1}{3}}$. For $q<L_{L}^{-1}$ the mode saturates at the pinning freqency $\bar{\omega}_{p}^{2} / \omega_{c}$. The length scale $L_{L}$ is set by matching the longitudinal stiffness to the pinning frequency, viz $\bar{\omega}_{p}=\omega_{L}\left(q=a / L_{L}\right) ;$ in either the screened or the unscreened limit $L_{L} \gg L_{T}$, so the finite- $\omega$ intercept in Fig.1 at $q \rightarrow 0$ is barely visible. In the opposite, large conductivity limit the dispersion becomes linear $\omega=\left(\Omega \bar{\omega}_{p} / \omega_{c}\right)(q a)$ but clearly because the mode is pushed to a finite frequency at $q=0$ the free-carriers must eventually become unimportant at long enough wavelengths. 


\section{Nonlinear DC response}

\subsection{Hall resistivity}

It is worth noting that one can obtain the Hall resistivity immediately from Eq. 2 [12]. We consider the Hall resistivity in the sliding state of a Wigner crystal. With a constant current density $j_{x}$ in the $x$-direction, the Hall resistivity relates $E_{y}^{e x t}$ to $j_{x}$ via $E_{y}^{e x t}=\rho_{y x} j_{x}$. We average the Fourier transformed form of Eq. 2 on both sides over disorder at a steady state under the condition that $u_{x} \neq 0$. Assuming isotropy, we readily have:

$$
\rho_{\mathrm{c}} E_{y}^{e x t}=\rho_{m} \omega_{c} \dot{u}_{x}
$$

Since $j_{x}=\rho_{\varepsilon} \dot{u}_{x}$, we have $\rho_{y x}=B / \rho_{c} e$. This argument is independent of the form of the disorder potential, and applies also to the linear response below threshold in the limit $\omega \rightarrow 0$, provided the WC polarisation current dominates the response. This provides further evidence that the arguments given by Kivelson et al.[17] for the "Hall insulator", characterised by a diverging longitudinal resistivity and constant Hall resistivity, apply in general to any insulating phase. Indeed experiments have observed a classical value of the Hall resistance [18].

\subsection{High-velocity perturbation theory}

At high velocities, the random potential provides only a small perturbation on the motion of the WC, and it is possible to develop a perturbation series for the velocity $\vec{v}$ of the WC in inverse powers of the electric field, similar to the methods applied to the vortex lattice[10] and CDW[19]. For the present, we assume $\sigma_{x x}=0$ so screening effects are neglected. Only the main results are given here, and details of the calculations are presented elsewhere.[12] The extra drag results from the excitation of modes in the WC lattice at the "washboard" frequency $\omega_{v}=\vec{G} \cdot \vec{v}$, and depend on whether or not the dispersion is overdamped at this frequency. The results for the overdamped case are given here.

In the absence of pinning, the solution of $\mathrm{Eq}$. (2) for the WC velocity is

$$
\frac{\rho_{c}}{\rho_{m}} \vec{E}^{e x t}=\left(\begin{array}{cc}
\gamma & -\omega_{c} \\
\omega_{c} & \gamma
\end{array}\right) \cdot \vec{v}
$$

and we then write the displacement as

$$
\vec{u}(\vec{r}, t)=\vec{v} t+\vec{x}(\vec{r}, t),
$$

where $\vec{x}$ represents the additional displacement due to disorder scattering. Calculating to the second order Born approximation, we obtain corrections to the high field conductivity of order $1 / \mathrm{E}$. For the resistivity, we find

$$
\rho_{x x}=\frac{\gamma \rho_{m}}{\rho_{c}^{2}}[1+\beta / v \gamma], \text { and, } \rho_{x y}=\frac{\omega_{c} \rho_{m}}{\rho_{c}^{2}}=B / \rho_{c} .
$$

As advertised, we find $\rho_{x y}$ unaffected by disorder. The parameter $\beta$ is proportional to the mean square fluctuation of the disorder potential. In a constant current $j=\rho_{\mathrm{c}} v$ along $\hat{x}$, Eq. (15) implies that the longitudinal electric field $E_{x}$ is

$$
E_{x}=\frac{\gamma \rho_{m}}{\rho_{c}^{2}} j+E_{T h}
$$

where the estimate of the threshold field $E_{T h}$ is

$$
E_{T h} \approx \beta \rho_{m} / \rho_{c} \approx a \frac{\rho_{m}}{\rho_{c}} \bar{\omega}_{p}^{2}
$$

Another quantity of interest is the strain in the WC induced by disorder. It is important for the validity of the elastic medium description that the local strains $\partial \vec{u} / \partial \vec{r}$ be small, else the lattice will tear. Thus we calculate

$$
E_{L}^{s}=\left\langle(\nabla \cdot \vec{x}(\vec{r}, t))^{2}\right\rangle, \text { and, } E_{T}^{s}=\left\langle(\nabla \times \vec{x}(\vec{r}, t))^{2}\right\rangle .
$$

They are respectively proportional to the elastic energies associated with the bulk and shear moduli. Because of the soft shear modulus, $E_{T}^{s}$ is the larger, and we find

$$
E_{T}^{s} \approx\left(\frac{a}{L_{T}}\right)^{2} \ln \left(\frac{\omega_{v} \gamma}{\Omega^{2} \alpha}\right)
$$

The argument of the logarithm is approximately $\left(\gamma / \omega_{c}\right)\left(\epsilon E / \rho_{c} \alpha\right)$. As in the two-dimensional CDW problem, the average strain diverges logarithmically at small velocities [20] and in consequence the lattice must break up above the threshold predicted by the elastic model, even if $L_{T} / a$ is very large. In strongly disordered systems close to $E_{T h}$, the current is likely carried mostly via defects in the lattice, as has been seen in numerical simulations of the vortex lattice [21]. 


\subsection{Screening effects close to threshold}

The effective damping appearing in Eq. (7) is very large at low temperatures, and will be important in the hydrodynamic limit when $\omega_{v} / \Omega \ll\left(\sigma_{x x} / \sigma_{o}\right)\left(a / L_{L}(E)\right)$, where $L_{L}(E)$ is the characteristic length scale for density fluctuations in the sliding state. This condition is inconsistent with the high-velocity limit discussed in the last section, but must be valid close enough to threshold. This regime is not accessible to a simple analytic treatment because the fluctuations in the local velocity are large. By balancing the dissipation $\gamma_{e f f} \rho_{m} \dot{u}_{L}$ against the excess field above threshold we get a rough estimate of the magnitude (though not the functional form) of the polarisation current of the sliding crystal:

$$
J_{W i g n e r}=\rho_{c} \dot{u}_{L} \approx \frac{\rho_{c}^{2}}{\rho_{m}} \frac{E-E_{T h}}{\gamma_{e f f}}=\sigma_{x x}\left(E-E_{T h}\right) .
$$

Consequently the nonlinear sliding conductance is comparable in magnitude to the linear "background" conductance, as in the case of CDW systems [22]. The hydrodynamic limit breaks down at a field $E_{x}$ which is roughly

$$
E_{x}-E_{T h} \approx\left(\rho_{c} / \epsilon\right)\left(a / L_{L}\right) \approx(\alpha)^{-\frac{2}{2}}\left(L_{T} / a\right) E_{T h}
$$

which can be a sizeable range only in a relatively clean system. Above this field, there will be a smooth crossover to the high-field regime treated in the previous section.

\section{Conclusions}

The experimental situation remains unclear, and a detailed comparison of our theoretical results to data is not practicable (for some attempts to estimate the correlation length see [11]), but we do have some general remarks. We mentioned in the introduction that the treatment of the WC as an elastic medium is most likely valid near a second order transition, when the amplitude of the WC is small i.e. $\Delta / \rho_{c} \ll 1$. Also, in Sec. 4.2 and Eq. (19), we established that the elastic description is reliable at large enough velocities (though of course small in comparison to the characteristic phonon frequency at the zone boundary: $\left.\omega_{v} \ll \alpha^{\frac{1}{2}} \Omega^{2} / \omega_{c}\right)$. Consequently, one might expect the most reliable comparison to experiment for electron-doped samples in the vicinity of $\nu=1 / 5$, where we believe that there is evidence for second-order character to the WC/FQHE transition.

The threshold field $E_{T h}$ has a local maximum just at the onset of the insulating phase either side of $\nu=1 / 5$ and decreases as the field is varied away $[4,8]$. We also note that the linear resistance $\rho_{x x}$ is activated in the insulating phase, with an activation energy which apparently vanishes continuously at the transition [8]. At a continuous transition, one expects a softening of the shear modulus $\mu$, and a consequent decrease in the correlation length $L_{T}$ which is proportional to $\mu / V$, where $V$ is a measure of the pinning potential of disorder. Since $E_{T h} \propto \mu / L_{T}^{2} \propto L_{T}^{-1}$, we then expect a rise in the threshold field on approach to the transition, as seen in experiment.

Further evidence for a continuous transition comes from low frequency capacitance measurements. In the limits $q \ll L_{L}^{-1}, \omega \ll \bar{\omega}_{p}^{2} / \omega_{c}$, the dielectric response of the pinned WC is proportional to $\bar{\omega}_{p}^{-1}[11]$. Li et al. [8] show that the capacitance drops to zero within the insulating phase, and in fact has a peak roughly corresponding to the minimum in $E_{T h}$.

Another feature of note is that the nonlinear conductance just above threshold indeed shows an activated temperature-dependence [5], consistent with our prediction in Eq. (20). Measured threshold fields in the range of $\left\langle 1 \mathrm{mV} / \mathrm{cm}\right.$ correspond roughly to $\left.L_{T} / a\right\rangle 10$, and so the conditions for both weak pinning and for screened motion (Eq. (21)) are satisfied.

At larger B, for $\nu<0.19$, both threshold field and pinning frequency appear to increase rapidly [3, 4, 7, 8], implying a decrease of $L_{T} / a$ to $\approx 1$ for $\nu<0.17$. One possibility is the failure of the elastic continuum model (which is of course implied by such a short correlation length) on account of the increase in amplitude of the WC. Transport in this regime would then be largely via dislocations [21]. (Note again that near $\nu=1 / 5$ evidence for collective transport can be found from interference phenomena at the "washboard" frequency $\omega_{v}$ [23]).

We conclude that there is quite good evidence for a WC phase whose nonlinear dynamics can be treated within a classical elastic medium desription, at least close to the onset of the insulating phase. At large magnetic fields, the behavior is presently enigmatic. Certainly, more attention needs to be paid to incorporating aspects of plastic flow.

Acknowledgements

We would like to thank Y.P.Li, M.A.Paalanen, D.C.Tsui and R.L.Willett for useful discussions, and for making available to us their results prior to publication.

\section{References}

[1] H. W. Jiang, R. L. Willett, H. L. Stormer, D. C. Tsui, L. N. Pfeiffer, and K. W. West, Phys. Rev. Lett. 65, 633 (1990). 
[2] V. J. Goldman, M. Santos, M. Shayegan, J. E. Cunningham, Phys. Rev. Lett. 65, 2189 (1990).

[3] F. I. B. Williams, P. A. Wright, R. G. Clark, E. Y. Andrei, G. Deville, D. C. Glattli, O. Probst, B. Etienne, C. Dorin, C. T. Foxon, and J. J. Harris, Phys. Rev. Lett. 66, 3285 (1991).

[4] Y. P. Li, T. Sajoto, L. W. Engel, D. C. Tsui, and M. Shayegan, Phys. Rev. Lett. 67, 1630 (1991).

[5] H. W. Jiang, H. L. Stormer, D. C. Tsui, L. N. Pfeiffer, and K. W. West, Phys. Rev. B 44, 8107 (1991).

[6] E.Y.Andrei, G.Deville, D.C.Glattli, F.I.B.Williams, E.Paris, and B.Etienne, Phys.Rev.Lett. 60, 2765 (1988); 62, $973(1989)$

[7] M.A.Paalanen, R.L.Willett, R.R.Ruel, K.W.West, L.N.Pfeiffer, D.J.Bishop, and P.B.Littlewood, Phys.Rev.B 45, 11342 (1992).

[8] Y.P.Li, D.C.Tsui, T.Sajoto, L.W.Engel, L.Xu, M.Santos, and M.Shayegan, unpublished.

[9] For a review on CDW systems, see, G. Grüner, Rev. Mod. Phys. 60, 1129 (1988).

[10] A. Schmid and W. Hauger, J. Low. Temp. Phys. 11, 667 (1973); A. I. Larkin and Yu. N. Ovchinnikov, Zh. Eksp. Teor. Fiz. 65, 1704 (1973) [Sov. Phys. JETP 38, 854 (1974)].

[11] B. G. A. Normand, P. B. Littlewood, and A. J. Millis, Phys. Rev. B 46, 3920 (1992); A. J. Millis and P. B. Littlewood, unpublished.

[12] X. Zhu, P. B. Littlewood, and A. J. Millis, unpublished.

[13] H. Fukuyama and P. A. Lee, Phys. Rev. B 18, 6245 (1978); H. Fukuyama, J. Phys. Soc. Japan. 41, 513 (1976); Surf. Sci. 58, 320 (1976).

[14] L. Bonsall and A. A. Maradudin, Phys. Rev. B. 15, 1959 (1977).

[15] R. Coté and A. MacDonald, Phys. Rev. Lett. 65, 2662 (1990); Phys. Rev. B 44, 8759 (1991).

[16] H. Fukuyama and P. A. Lee, Phys. Rev. B 17, 535, (1978); P. A. Lee and H. Fukuyama, ibid, 542 (1978); P. A. Lee and T. M. Rice, Phys. Rev. B 19, 3970 (1979); T. M. Rice, P. A. Lee, and M. C. Cross, Phys. Rev. B 20, 1345 (1979).

[17] S. C. Zhang, S. Kivelson, and D. H. Lee, Phys. Rev. Lett. 69, 1252 (1992); S. Kivelson, D. H. Lee, and S. C. Zhang, Phys. Rev. B 46, 2223 (1992); O. Viehweger and K. B. Efetov, Phys. Rev. B 44, 1168 (1991).

[18] V. J. Goldman, J. K. Wang, B. Su, and M. Shayegan, Phys. Rev. Lett. 70, 647 (1993); T. Sajoto, Y. P. Li, L. W. Engel, D. C. Tsui, and M. Shayegan, Phys. Rev. Lett. 70, 2321 (1993).

[19] L. Sneddon, M. C. Cross, and D. S. Fisher, Phys. Rev. Lett. 49, 292 (1982); L. Sneddon, Phys. Rev. B 29, 719 , 725 (1984).

[20] S. N. Coppersmith, Phys. Rev. Lett. 65, 1044 (1990); S. N. Coppersmith and A. J. Millis, Phys. Rev. B 44, 7799 (1991).

[21] A. C. Shi and A. J. Berlinsky, Phys. Rev. Lett. 67, 1926 (1991).

[22] P.B.Littlewood, Phys.Rev.B 36,3108 (1987); Solid State Commun. 65, 1347 (1988).

[23] Y.P.Li, D.C.Tsui, T.Sajoto, L.W.Engel, L.Xu, M.Santos, and M.Shayegan, unpublished. 